\begin{tabular}{|l|l|l|l|l|}
\hline Revista Clío América & ISSN: 1909-941X & Vol.9 & No. 17 & Enero - Junio de 2015 \\
\hline
\end{tabular}

\title{
La responsabilidad social empresarial (RSE) de los pequeños distribuidores minoristas
}

\author{
Corporate social responsibility (CSR) of small retailers
}

Resumen: Se analiza la aplicación de la Responsabilidad Social Empresarial (RSE) a los pequeños distribuidores minoristas de bienes de consumo en una ciudad de Colombia (Medellín). Partiendo de un trabajo empírico en la región mencionada, se examinaron elementos que generan posicionamiento a estos comerciantes frente a la amenaza de los grandes distribuidores minoristas (comercio moderno de grandes superficies) incluyendo el constructo de la RSE y algunos de sus componentes que producen reputación a estas personas frente a dichos competidores.

Se examina en la literatura la forma en que el concepto de la RSE se asocia a las pequeñas empresas, la relación de esta variable con la distribución minorista estudiando los principales resultados de la investigación desarrollada con tenderos de barrio (pequeños distribuidores minoristas) en Medellín durante el año 2011, en la que intervinieron igualmente clientes de estos comerciantes y algunos de sus proveedores (fabricantes, distribuidores mayoristas).

Se expone igualmente la metodología cualitativa utilizada en la investigación basada en la teoría fundamentada (Grounded Theory) complementada con la Etnografía. La recolección de información fue realizada a través de grupos focales, observación no participante y entrevistas individuales en profundidad semiestructuradas. Finalmente se realiza una discusión acerca de los principales hallazgos lo mismo que sus conclusiones más relevantes.

Palabras clave: Responsabilidad Social Empresarial, Distribución minorista, Pequeñas y medianas empresas.

JEL: L25, L81, M14, M31.

Abstract: The implementation of Corporate Social Responsibility (CSR) to small retailers of consumer goods in a city of Colombia (Medellin) is analyzed. From an empirical investigation it is studied some elements that generate positioning for these traders face the threat of large retailers (supermarkets with modern trade) including the construct of CSR and some components that produce reputation for these people against those competitors.

It is discussed in the literature how the concept of CSR is associated with small businesses, the relationship between this variable and retailing studying the main results of research carried out with neighborhood shopkeepers (small retailers) in Medellin during 2011
Juan Carlos Sanclemente-Téllez $\mathrm{PhD}$ en Administración Universidad EAFIT, Colombia jsanclem@eafit.edu.co

Tipología:

Artículo de Investigación Científica y Tecnológica

Fecha de Recibido:

Enero 26 de 2015

Fecha de Aceptación: Abril 07 de 2015

Para citar este artículo:

Sanclemente, T. J. (2015). La responsabilidad social empresarial (RSE) de los pequeños distribuidores minoristas, Clío América, 9 (17), 42 - 56 
in which also intervened customers of these dealers and some of its suppliers (manufacturers, wholesale distributors).

Qualitative research methodology based on Grounded Theory supplemented with Ethnography is also exposed. Data collection was conducted through individual semi-structured depth interviews, focus groups and non- participant observation. Finally, a discussion of the main findings as well as their most relevant conclusions is made.

Keywords: Corporate Social Responsibility, Retailing, Small and Medium Enterprises (SMEs).

\section{Introducción}

Se ha llegado a adoptar la idea en los últimos años, de que las empresas y todo tipo de organizaciones tienen responsabilidades sociales, llevando esta situación tanto a académicos como a los hombres de negocios, a manifestar intereses y preocupaciones variadas por el tema que se ha denominado como RSE (Responsabilidad Social Empresarial). Este concepto ha surgido tanto como un importante constructo académico como un elemento de presión en la agenda de todo tipo de empresas, particularmente las grandes organizaciones y los conglomerados. A pesar de tantos años de incesante debate académico en cuanto a la RSE, es posible decir con certeza que no existe una definición universalmente aceptada del término y que tan solo las más grandes empresas parecen haber encontrado, a pesar de no contar la RSE con sus elementos constitutivos establecidos de manera concreta, un terreno común sobre el que han construido elaboradas prácticas y políticas sobre el tema. La RSE ha recibido entonces considerable atención en los últimos años pero tan solo muy pocos estudios han sido conducidos teniendo en cuenta las responsabilidades sociales de las pequeñas empresas, ¿será que ellas están menos concernidas socialmente que las grandes empresas o las corporaciones? ¿Serán ellas incapaces de invertir determinados montos de tiempo y de dinero en este tema tan primordial hoy en día en los negocios? ¿Cómo operaría este tema en el caso de la distribución minorista y más específicamente en el caso de los pequeños distribuidores minoristas? Este estudio trata estas y otras preguntas acerca de las perspectivas de los tenderos de barrio (que pertenecen a esta categoría de comercio) acerca de sus responsabilidades en la sociedad.

\section{La responsabilidad social empresarial de las pequeñas empresas}

Tradicionalmente, la responsabilidad social empresarial ha sido un tema del sector corporativo (Jenkins, 2006), pero cada vez más se reconoce la creciente significación que el sector de las pequeñas y medianas empresas presenta hacia el énfasis en el impacto social y medioambiental y que se puede ilustrar por un creciente número de iniciativas que involucran a las pymes en la agenda de la RSE en los ámbitos local, nacional e internacional. En efecto, se constata que el tema de la RSE ha sido suficientemente investigado para las grandes compañías, pero las pymes han recibido más bien poca atención en este sentido (Jenkins, 2006; Lepoutre \& Heene, 2006; Perrini, 2006; Russo \& Tencati, 2008; Spence, 2007; Murillo \& Lozano, 2006; Thompson \& Smith, 1991). La importancia de las empresas pequeñas y medianas en la economía de todos los países ha incidido en que si bien "la RSE nació asociada a las grandes empresas multinacionales, con el paso del tiempo se ha visto la necesidad de trasladar el concepto a las pymes" (0brsc, ${ }^{1} 2005$, p. 13).

Las pequeñas y medianas empresas son parte fundamental de toda economía y es bien conocida su contribución a la creación de empleo y al PIB de un territorio (Royo, 2007, p. 445). La mejora de la competitividad empresarial de este colectivo de

1. Observatorio de Responsabilidad Social Corporativa, España, 2005. 
empresas pasa a ser uno de los elementos claves para desarrollar tanto las economías como las sociedades latinoamericanas (Vives et al., 2005), pero se debe ahondar aún más en la relación entre este tipo de empresas y las actividades que se deben llevar a cabo respecto a la RSE, puesto que muy raramente este grupo de empresas utiliza el lenguaje de la RSE para describir sus actividades en este campo; por el contrario, las estrategias informales en responsabilidad social juegan un papel importante en ellas (Russo \& Tencati, 2008, p. 339).

Otros se refieren a la distinción que se debe hacer en el análisis de la teoría referente a la RSE, entre las grandes empresas y las pymes: la investigación específica en las grandes organizaciones debería estar basada en la teoría de los stakeholders, mientras que la investigación de la RSE en las pequeñas y medianas empresas debería estar basada en el concepto de capital social (Perrini, 2006, p. 305)2. De igual manera, otros autores mencionan que existen muchos campos que tienen relación con la RSE y que también se puede llegar a considerar como un objeto de estudio "multidisciplinario y ecléctico" (Perrini, 2006; Maignan \& Ferrell, 2004), en el que no se puede dejar a un lado a los gerentes que conducen una empresa (sean los mismos propietarios o simplemente empleados), puesto que su orientación hacia el tema de la responsabilidad social en su gestión es muy importante (Burton \& Goldsby, 2009, p. 88). Los campos de actividad en los que se pueden desenvolver las pequeñas y medianas empresas en asuntos sociales o de RSE, la orientación que ellas quieran darle a la acción en este sentido y las estrategias (formales 0 informales) que llevan a cabo a diario son poco exploradas en el campo de la literatura académica (Burton \& Goldsby, 2009). Algunos de los debates conceptuales que se han dado previamente exhiben como argumento que las pymes presenten menos expectativas frente al tema de la RSE, comparadas con las grandes compañías, puesto que su actividad tiene menor impacto y menor poder que estas últimas en la sociedad y "no es que no tengan responsabilidad social, sino que deben simplemente ser responsables de entregar a la

2. El capital social se refiere a "las conexiones entre individuos -redes sociales y las normas de reciprocidad y confianza que surgen de ellos" (Perrini, 2006, p. 308). sociedad productos y servicios de calidad de manera que no hagan daño a ninguna de las partes" (Van Auken \& Ireland, 1982, p. 2).

Arlow \& Ackelsberg (1991, p. 161) argumentan que “al tener menos poder económico y recursos que las grandes firmas, las organizaciones pequeñas pueden hacer poco más que reconocer la importancia de la responsabilidad social." A pesar de estas limitaciones, se puede percatar un papel de responsabilidad social en los pequeños negocios, puesto que "los asuntos sociales que afectan a estas empresas son probablemente pequeños en alcance" (p. 162), por lo que se pueden concentrar en cuestiones locales y de participación comunitaria. A su turno, Thompson \& Smith (1991, p. 39) mencionan que existen oportunidades limitadas para las pequeñas empresas en lo referente al ejercicio de sus responsabilidades sociales y que ellas se han ganado la reputación de ser menos capaces que las grandes para cumplir tales obligaciones. A pesar de ello, insinúan que "las acciones sociales de las pequeñas empresas están únicamente limitadas por la imaginación de sus gerentes o propietarios" (p. 39). Esta última aseveración deja ver que, en el campo de estas empresas, existe mucha imaginación y creatividad para abordar los asuntos sociales que hacen parte de la RSE. De igual manera se corrobora otra afirmación, en el sentido de que estas empresas pueden llevar a cabo acciones de RSE de manera informal sin que necesariamente hagan parte de la estrategia del negocio. Este es un punto muy interesante en la investigación, relacionada con el tendero de barrio, ya que esta persona puede llevar a cabo en el marco de su negocio diversas actividades de carácter social que brinden un apoyo a la comunidad y hacerlo "desprevenidamente", o bien, "ingeniarse" actividades a favor de la misma con el ánimo de cultivar una buena imagen entre sus clientes con el fin, por ejemplo, de que estos le compren más a menudo.

En el campo de las investigaciones empíricas, éstas muestran que en efecto las pequeñas empresas se sienten aludidas con los asuntos atinentes a la RSE (así sea que ejerzan sus acciones de manera formal 0 informal y con conocimiento o desconocimiento del tema y de su alcance). Algunos estudios realizados en Norteamérica (Chrisman \& Archer, 1984; Chrisman $\&$ Fry, 1982; Wilson, 1980) y otros llevados a cabo en 
Latinoamérica efectuando contrastación con pymes europeas (Vives et al., 2005), en Colombia (CCRE, 2006) y en Antioquia (Giraldo, 2008) demuestran esta aseveración.

Teniendo presente que, aunque las pequeñas empresas puedan experimentar ciertas "limitaciones" en el desarrollo de actividades y estrategias dirigidas a la RSE entre sus grupos de interés, de todas maneras llevan a cabo acciones que se inscriben en el campo de acción de la misma (de hecho, en el marco de la investigación se pretendió encontrar esas acciones que de manera formal o informal llevaban a cabo los pequeños empresarios tenderos de barrio y la manera en que las mismas eran percibidas por los consumidores 0 clientes que hacen parte de sus "grupos de interés"). En este sentido, Wilson (1980, p. 20) realizó, en compañía de sus estudiantes, una investigación de carácter fenomenológico (enfoque de corte cualitativo que utiliza un análisis de protocolo y que pretende "ir a los fenómenos como tales") en el área metropolitana de Los Ángeles, en la que se entrevistó (planteando una sola pregunta básica: ¿cómo ve usted sus responsabilidades frente a la sociedad?) a 180 propietarios o gerentes de pequeños negocios con menos de 25 empleados, efectuó clústeres de temas a partir de las afirmaciones de los empresarios y con base en estos encontró al menos cinco categorías significativas de acciones de RSE: responsabilidades hacia el consumidor, responsabilidades con los empleados, ética, responsabilidad hacia la comunidad, ganancias o beneficios.

En lo que toca con la investigación latinoamericana (Vives et al., 2005), la cual abarca a Colombia, y la investigación concretamente colombiana (CCRE, 2006), se observa en la primera de ellas que para las pymes prima la vertiente interna de la RSE (la dirigida a los empleados 0 trabajadores y al buen funcionamiento de la empresa) sobre los aspectos externos (los dirigidos a la comunidad) y los medioambientales. En el caso colombiano, no se discriminan concreta y completamente las actividades de las pymes en la RSE pero, en términos generales, las empresas en nuestro país enfocan sus acciones en este campo en primer lugar hacia los empleados $y$ en segundo hacia el "trabajo por la comunidad" (CCRE, 2006, p. 19).
Pasemos ahora a mencionar algunos elementos atinentes a la RSE desarrollados por las empresas que pertenecen al campo de la distribución minorista, al cual se vincula el comerciante analizado. A continuación se hace una breve referencia a la responsabilidad social empresarial asociada con la distribución minorista que en ningún momento se pretende exhaustiva.

\section{La responsabilidad social empresarial en la distribución minorista}

Aunque la preocupación por el tema de la RSE de manera habitual ha sido ligada a las grandes empresas o al sector corporativo, las empresas dedicadas a la distribución comercial o retailing han advertido la importancia estratégica de este tema en su gestión y han incorporado dichas reflexiones en sus prácticas comerciales cotidianas. A continuación se realiza un breve recuento de la literatura existente en el tema de la RSE vinculada al sector minorista.

Wagner, Bicen \& Hall (2008, p. 124) mencionan que “la preocupación pública y la cobertura de los medios de comunicación acerca de la RSC en la industria de la distribución minorista ${ }^{3}$ se ha incrementado notablemente en los últimos años. En consecuencia, los distribuidores minoristas frecuentemente se esfuerzan por demostrar comportamientos socialmente responsables para ser percibidos como buenos ciudadanos corporativos".

Varios estudios han analizado la influencia que tienen las acciones de RSE desplegadas por las empresas sobre las percepciones y sobre el comportamiento de los consumidores (Sen \& Bhattacharya, 2001; Bhattacharya \& Sen, 2004; Mohr \& Webb, 2005; Marín, Ruiz \& Rubio, 2009; Marín y Ruiz, 2008) y algunos otros han investigado en forma específica la manera en que los consumidores perciben la responsabilidad social de los distribuidores minoristas (Lichtenstein, Drumwright \& Braig, 2004; Maignan, 2001), ubicándola, inclusive, dentro de lo que se ha denominado cause-related marketing 0 , en términos generales, acciones de mercadeo con una dimensión social (Maignan, 2001, p. 57; Maignan \& Ferrell, 2004, p. 3; Brown \& Dacin, 1997; Handelman \& Arnold, 1999, p. 33).

3. Retailing: distribución minorista, venta al por menor, venta al detal 
Es importante destacar que los estudios relacionados con las percepciones de los consumidores (y sus evaluaciones de las empresas en lo relativo a la RSE), y con las mismas acciones de RSE llevadas a cabo por los grandes distribuidores minoristas, han sido la norma, mientras que los consagrados a los pequeños empresarios minoristas ( 0 a las percepciones de sus clientes) constituyen la excepción.

En último lugar, Whysall (2000b) realiza un interesante análisis de los principales asuntos éticos en el campo del retailing, para lo cual se apoya en la teoría de los stakeholders, análisis que igualmente puede efectuarse con los pequeños comerciantes que también tienen grupos de interés relacionados con sus actividades comerciales diarias.

Para concluir esta parte relacionada con la RSE en el campo de la distribución minorista, se encuentra que los autores que han realizado una intensa y extensa revisión de literatura de los temas que pudieran tocar a los pequeños empresarios de la distribución minorista, confirman que este campo de estudio ha sido ignorado en contextos amplios y que en ocasiones se ha confundido dentro de la gran masa de investigaciones y estudios en retailing (Runyan \& Droge, 2008, p. 77).

\section{Metodología}

El enfoque metodológico utilizado para la investigación que sirve de apoyo para este artículo fue el cualitativo. Este procedimiento tuvo su soporte en el método de la teoría fundamentada (Grounded Theory, Corbin \& Strauss, 1990; Glaser \& Strauss, 1967), de carácter exploratorio e interpretativo, tratando de entender y encontrar significado a la situación estudiada. Esto supone fabricar una teoría que va surgiendo con los datos recolectados y que se encuentra debidamente contextualizada, tomando como testimonio esencial las contingencias, las experiencias y los puntos de vista de los actores que conformaron la unidad de análisis, e interaccionando en todo momento con el marco teórico. El método complementario escogido fue la Etnografía, asumida como una directriz metodológica que coloca todo el énfasis en el trabajo de campo, el contacto directo y la observación de los actores que se analizan, en el entorno natural en el que llevan a cabo sus actividades y durante un lapso de tiempo relativamente extenso. Las dos estrategias metodológicas (teoría fundamentada y etnografía) combinadas, se han manifestado como poseedoras del potencial para poder brindar un detallado entendimiento de muchos fenómenos del mercado (Pettigrew, 2000). De acuerdo al enfoque cualitativo del estudio y a las técnicas normalmente empleadas en los métodos escogidos, se emplearon las entrevistas en profundidad, los grupos focales y la observación no participante. Las entrevistas fueron realizadas a 18 tenderos de barrio (para los seis estratos socio económicos) y a sus principales proveedores (dos fabricantes y dos distribuidores mayoristas), los grupos focales se ejecutaron con los clientes de estos comerciantes (72 personas en total para todos los estratos). Los tenderos de barrio fueron abordados con preguntas tales como: "¿Cómo ve usted su responsabilidad ante la sociedad (o la comunidad)?" y “¿Cuáles son sus principales responsabilidades sociales en la comunidad?" Para los proveedores y clientes se utilizaron como preguntas iniciales: “'Cómo ven ustedes la responsabilidad social del tendero de barrio ante la sociedad (o la comunidad)?” y “¿cuáles son las principales responsabilidades sociales del tendero de barrio en ella?" (Sanclemente, 2012b, 2014).

Se utiliza pues, una metodología que es netamente de "descubrimiento" (la teoría fundamentada) y no de justificación, y por lo tanto las categorías (0 divisiones de la RSE) que se presentan en la investigación proceden de una indagación directa con los elementos que hacen parte de la unidad de análisis de la investigación y van emergiendo a partir de los datos que se van recolectando (característica propia de la teoría fundamentada) y la conceptualización de las mismas ha sido desarrollada con el apoyo de herramientas de análisis de datos cualitativos (Atlas T.i.) y no provienen de una teoría previamente establecida (aunque se realiza la revisión de literatura correspondiente, tanto de la RSE en las pymes como en las empresas de distribución minorista). Suddaby (2006, p. 634) menciona que la "teoría fundamentada no es una excusa para ignorar la literatura", y que, por lo tanto, deben existir dentro del método tanto preguntas de investigación como literatura previa con las cuales el investigador enfrenta el estudio. 


\section{Responsabilidad social empresarial (RSE) del tendero de barrio}

En este apartado se elabora una descripción de los distintos tipos de responsabilidad social identificados en los tenderos de barrio y que son producto de la investigación realizada no solo con estos comerciantes sino igualmente con sus clientes y proveedores.

La conceptualización de este constructo (RSE) en el caso del tendero de barrio y la aparición de las correspondientes categorías y subcategorías fue realizada con el apoyo del programa informático de manejo de datos cualitativos Atlas t.i. ${ }^{\circledR}$, y siguiendo la verbalización suministrada por los informantes (parámetro de funcionamiento de la teoría fundamentada) ${ }^{4}$.

\section{Responsabilidad social empresarial económica}

Entendida, de acuerdo a Sanclemente (2012b, 2014), como la responsabilidad que tienen estos comerciantes de buscar que su actividad sea rentable conservando un elevado nivel de efectividad operativa y permitiendo que ella le procure ingresos y le asegure sostenibilidad. Un comercio de esta índole, productivo y floreciente, solo puede permanecer en una sociedad 0 ambiente saludable (principalmente desde el punto de vista económico); y asimismo, un tendero, adicional a garantizar su propia permanencia, puede verse vinculado con proporcionar oportunidades de empleo a otros individuos de su barrio, distintos a su familia e igualmente fomentar la adquisición de bienes a empresarios de distintos sectores económicos que igualmente pertenecen al vecindario en el que él tiene su negocio comercial.

\section{Rentabilidad}

Que la actividad que lleva a cabo el tendero de barrio le genere un margen o una remuneración representa-

\footnotetext{
4. De igual manera y por cuestiones de requisitos de especificaciones para est artículo (principalmente en cuanto a extensión), no se muestran los verbatims expresados por todos los informantes y que respaldan todas y cada una de las clasificaciones realizadas para el constructo respectivo, estos quedan a disposición de los lectores del presente documento y pueden ser suministrados bajo demanda al igual que el mapa conceptual producto del manejo de la información que permitió la posterior conceptualización.
}

tiva a su esfuerzo y que le permita no solo subsistir diariamente con su familia sino igualmente a tener abierto el negocio durante el tiempo. Lo básico, en términos económicos, es que el negocio produzca más ganancias que pérdidas y que se proyecte hacia el futuro. Algunos excedentes de la actividad básica comercial del tendero podrán ser utilizados para atender otros tipos de responsabilidad social empresarial (por ejemplo: voluntaria con donaciones).

\section{Fiado}

Se trata de un concepto que hace referencia al dinero que los clientes quedan debiendo al tendero de barrio cuando hacen una compra y para pagar esa suma en un plazo acordado futuro. También se menciona el denominado "monedero virtual", situación en la cual los clientes quedan debiendo en dinero una parte de la compra porque "no les alcanzó".

En el marco de este análisis se toma como el préstamo 0 crédito que el tendero de barrio otorga a sus clientes por considerarlos "dignos de confianza" y personas honestas. El tendero "confía" en los demás y otorga esta especie de microcrédito mediante el otorgamiento de plazos razonables para cubrir la deuda (el pago en dinero). El inadecuado manejo de los flujos de dinero: ingreso por ventas y egresos por pagos a proveedores, puede comprometer la existencia del negocio de la tienda en un muy corto plazo (fiar de manera persistente, no cobrar y tener que cumplir con obligaciones de pagos a terceros con oportunidad).

La inclusión del fiado dentro de la RSE económica tiene relación con la necesaria rentabilidad que debe buscar el tendero de barrio para poder sacar adelante su negocio, en este caso, le corresponde gestionar muy bien el aspecto del crédito a sus clientes para que este no tenga una incidencia desfavorable en el manejo de sus ingresos y egresos, comprometiendo su negocio (es más bien un tipo de responsabilidad que tiene este comerciante consigo mismo). Las opiniones de los informantes en este sentido, se muestran inclinadas hacia una crítica del fiado, puesto que su mal manejo expone la existencia del negocio y es responsabilidad del tendero garantizar su permanencia en el tiempo. La otra manera de ver el fiado, es ubicándolo dentro de la RSE comercial 
con los clientes, aspecto que se trata más adelante y que opera como un servicio (crédito informal) al brindarle formas de pago finamente ajustadas a la necesidad y realidades económicas de sus clientes.

\section{Responsabilidad social empresarial ambiental}

Consiste en el compromiso de este comerciante para con la atención, conservación y defensa del entorno en que desarrolla sus actividades mercantiles.

\section{Aseo}

Involucra la limpieza no solo del propio negocio, sino igualmente de la calle en la que se encuentra ubicado.

\section{Reciclaje}

Propender por la reutilización de materiales o de productos que ya han sido utilizados para producir productos nuevos, lo que incide de suyo en forma directa en la adecuada y óptima utilización de los recursos naturales. Los tenderos de barrio colaboran con este proceso cuando extraen de sus negocios y entregan a los "recicladores" estos materiales.

\section{Manipulación de alimentos}

Tener en cuenta la higiene, las fechas de vencimiento y el daño que los productos desechados puedan tener sobre el entorno (tanto a las personas como a la naturaleza). Una efectiva acomodación de los productos en las estanterías del establecimiento y el manejo directo que el tendero hace de los mismos conserva la limpieza y evita la mezcla de productos que no se pueden combinar (ejemplo: detergentes con comida).

Las opiniones de los informantes en cuanto a este elemento y al siguiente (contaminación) dejan ver sobre todo situaciones 0 acciones "ideales" que buscan los demás que realicen los tenderos, pero que no necesariamente estos llevan a cabo (verificado mediante la observación del investigador en el terreno).
El no cumplimiento de estas consideraciones "ambientales" por parte del tendero de barrio, lo lleva a tener en las percepciones de la gente, un concepto de "irresponsable", teniendo ello unas implicaciones negativas para la gestión de su negocio.

\section{Evitar contaminación}

Se refiere a no generar ruido ambiental, no ensuciar el medio ambiente, no alterar las condiciones puras y físicas de ciertos productos al mezclarlos o combinarlos, ahorrar energía, etc. Si bien en este caso en particular se presentan algunas opiniones muy relacionadas con el "deber ser" o con la "obligación" que tiene el tendero en este elemento, en la observación en terreno se advierte que algunos de ellos lo cumplen y otros no. Se encuentran igualmente algunas críticas en cuanto al manejo de la energía que al ser indebido puede afectar la imagen de "responsabilidad" que tiene este comerciante, aunque se resalta también la intervención o el apoyo que pudieran brindar los proveedores y el mismo gobierno, tanto en el sentido de los aparatos viejos como de los empaques de los productos respectivamente. En el campo se recogieron inquietudes y opiniones de los tenderos en cuanto a que los proveedores no colaboran con el tema de costos relacionados con el consumo de energía, cuando lo que se está promocionando son justamente sus productos.

\section{Responsabilidad Social Empresarial Ética}

Consiste en realizar acciones correctas, justas y equitativas procurando no dañar al prójimo. Al encontrarse en interacción con los demás, tener el pleno reconocimiento y respeto de determinados imperativos morales y éticos adoptados por la comunidad en la que se desenvuelve.

\section{Honesto}

El tendero de barrio como persona proba, honrada, recta y razonable. Procurarles el bien a los demás y en especial a todas aquellas personas con las que interactúa a diario. Adquirir buenos suministros, de fuente legal y no engañar a sus clientes con productos que no son originales. No mentir ni burlarse 
de los clientes con las medidas y los pesos de los mismos productos.

En ocasiones incluso, el tendero mismo asume ciertas "cargas" en cuanto al costo del dinero en el tiempo cuando fía, puesto que él mismo asume que es una muestra de honradez no cobrar precios distintos (eventualmente cargando intereses por crédito) para aquellas personas que pagan de contado y para aquellas que recurren al "fiado". Se evidencia que este comerciante desconoce en muchas ocasiones cuánto gana y cuánto pierde en este tipo de operación puesto que desconoce el alcance y las implicaciones del manejo del dinero a través del tiempo. Como se ha visto en el "fiado" desde el punto de vista de la responsabilidad social económica, y como se verá en la comercial, se utiliza este mecanismo más como un argumento para poder atender mejor a sus clientes y brindarles más comodidades tan solo con el objetivo de no perderlos como clientes y de cierta forma tener un ingreso asegurado (así sea incierto).

\section{Justo}

Equilibrado, exacto, íntegro, recto, equitativo y preciso frente a la obligación de entregar a la gente bienes o servicios producto de su actividad en compensación por un dinero recibido de parte de esa gente. No aprovechar las eventuales circunstancias que puedan experimentar sus clientes para establecer cobros inadecuados por las mercancías que vende.

\section{Influir sobre el entorno}

El comportamiento o la conducta diaria del tendero en su negocio tienen una influencia directa sobre la comunidad al "dar ejemplo" cotidianamente con su actividad y la manera de actuar, en especial a la población infantil y a los jóvenes. La forma en que atiende directamente su negocio y las cosas que hace o deja de hacer, o permite hacer en su establecimiento, son una muestra clara de valores y principios que influyen sobre su entorno más próximo, el barrio y sus habitantes.
Respeto

Reconocer a los demás, valorarlos, considerar a alguien como de valor. Tener consideración, deferencia y atención con las personas de la comunidad.

\section{Responsabilidad social empresarial legal}

Se refiere al respeto y obediencia de la ley, en cuanto a desenvolverse de forma congruente y razonable con las normas o preceptos reglamentarios en vigor y de acuerdo con las entidades que los emiten.

\section{Cumplimiento obligaciones contractuales}

Pagar cumplidamente los servicios públicos (necesarios para el funcionamiento del negocio o del local en el que opera), pagar con oportunidad las obligaciones directas con el Estado (impuestos nacionales, impuestos locales, derechos y matrículas y registros mercantiles, etc.). Incluye el respeto de la legislación particular y específica al tipo de negocio, promulgada por el Estado y realizada por entes como las Cámaras de Comercio y las Secretarías de Hacienda y de Gobierno municipales o departamentales, al igual que las obligaciones que se tienen con empleados, clientes y proveedores. Respecto a los empleados, y a ellos mismos como comerciantes y empresarios, es importante destacar el pago de salarios y los pagos adicionales por las contribuciones de salud y pensiones que en la mayoría de los casos no se dan, puesto que gran parte de estas personas operan en la informalidad, como se ha expresado ya previamente.

\section{Alimentos certificados}

Respecto a la elaboración de los productos que se fabrican directamente por el tendero de barrio 0 que comercializa ya fabricados, se requieren los permisos respectivos de las autoridades de sanidad, de control de fabricación de alimentos, etc., por ejemplo: del Invima.

\section{Formalización/registro del negocio}

Se trata de revestir la actividad comercial de tendero de barrio de todos aquellos requisitos legales para 
poder operar conforme a la ley y a las costumbres en el país. En esencia, se trata de que los negocios estén debidamente registrados ante las instancias públicas que tengan relación con su actividad y que se incorporen al flujo económico, en especial mediante el pago de impuestos. Registrar el negocio hace parte de la formalización; es preciso hacerlo ante los entes reguladores y controladores (por ejemplo: Secretaría de Gobierno, Cámara de Comercio, etc.).

\section{Responsabilidad social empresarial comercial (llevada a cabo con los clientes y con los proveedores)}

Diariamente los tenderos de barrio interactúan con clientes y proveedores y deben atender las necesidades de los primeros y desarrollar acuerdos y relaciones con los segundos. El respeto de estas interacciones y relaciones constituye este tipo de responsabilidad social empresarial ${ }^{5}$.

\section{Responsabilidad social empresarial comercial con los clientes}

Handelman \& Arnold (1999) y Rallapalli, Vitell \& Szeinbach (2000) plantean en cuanto a las relaciones comerciales con los clientes, algunas variables vinculadas con precio, producto, marca, surtidos y ubicación.

\section{Relaciones con los clientes}

Mantener buen trato y buena atención con las personas que compran en la tienda de barrio, esforzarse por convertir a los consumidores en clientes (pasar del campo de las transacciones al de las relaciones). El largo plazo se involucra aquí pues las visitas de la comunidad al negocio ya no son esporádicas sino que son continuas y permanentes.

5. Wilson (1980), encuentra en su investigación que si bien muchos de los propietarios entrevistados mencionan este aspecto como responsabilidad social, en muchos de los relatos de estas personas se advierte que lo hacen para que se propicie la recompra, se incremente el número de clientes y las ganancias aumenten en el largo plazo.

\section{Precios}

El costo del producto para el consumidor o para el cliente debe ser justo, exacto, equilibrado, competitivo y razonable. Un precio adecuado para el cliente hace que el tendero sea responsable con la clientela en términos generales. No se debe cobrar en exceso en ningún momento.

\section{Producto}

La calidad del producto y la marca, que pertenecen en la mayoría de los casos a los proveedores fabricantes, son aspectos esenciales para que el tendero de barrio responda a las expectativas de los consumidores y de los clientes.

\section{Satisfacción}

Los clientes siempre evalúan los resultados que obtienen con los productos que compran y la manera en que son atendidos por el tendero, respecto a las expectativas que tienen de dichos productos y de dicha atención. Si el desempeño de los productos o de la atención es menor que sus expectativas, el cliente se siente insatisfecho e incluso decepcionado. Si el desempeño cumple con las expectativas, el cliente se encuentra satisfecho y si dicho desempeño supera ampliamente lo esperado, el cliente se considera altamente satisfecho e inclusive encantado y "deleitado". El tendero busca, en todos los casos, satisfacer a su clientela no solo con los productos de calidad que ofrece sino igualmente con la interacción y el proceso de relación que sostiene con ella. Aunque en algunos casos estos comerciantes se expresan con ciertas frases acerca del ideal de la satisfacción ("debo brindarles 'x cosa` para satisfacerlos), en el terreno se observa que efectivamente se esmeran por llevarlo a cabo.

\section{Servicio}

Las respectivas actividades no solo tienen que ver con la actividad comercial, sino también con cosas adicionales. Domicilio, empaque adecuado de productos, buen trato con las personas, colaborarles con situaciones personales (cuidado de niños, de casas, 
guardar paquetes, brindar información, etc.), tener un horario adecuado a la clientela y extenso, etc.

\section{Fiado}

Las propiedades y dimensiones de esta subcategoría ya fueron definidas previamente cuando se hizo referencia a la RSE económica. Desde el punto de vista de asociarlo con la RSE comercial con los clientes se destaca el hecho de que este tipo de crédito informal opera más como un elemento de atención por parte del tendero hacia esas personas y, que en la lógica comercial que maneja este comerciante, se trata de un sistema basado esencialmente en la confianza y en la palabra, no tiene contraprestación en bienes 0 prendas ni papeles firmados que garanticen al tendero el retorno del monto prestado ni tampoco se incluye el cobro de intereses. Este tipo de crédito funciona como una especie de programa de fidelidad: una vez que un cliente utiliza este tipo de servicio, existen fuertes motivaciones para que continúe comprando en esa tienda. En el razonamiento comercial que realiza el tendero, esto operaría como una especie de sentido de reciprocidad de sus clientes para con él.

\section{Responsabilidad social empresarial comercial con los proveedores}

Las relaciones con proveedores por parte de determinados clientes fue propuesta por Rapalli, Vitell \& Szeinbach (2000).

\section{Boca-oreja/voz-a-voz}

Es la transmisión de información a terceras personas (comunidad) por parte de los tenderos de barrio acerca del desempeño de los proveedores en su negocio. Hablar (bien o mal) de los productos de los proveedores, de sus representantes, de las marcas, del nivel de atención y de servicio que le prestan al comerciante.

\section{Acuerdos comerciales}

Cumplir con los pagos y con las condiciones de los pactos de negociación que efectúan periódicamente los proveedores con los tenderos de barrio. Pagarles y respetar plazos de entrega y de cobro de las mercancías que se intercambian.

\section{Responsabilidad social empresarial voluntaria}

Siguiendo a Sanclemente (2012b, 2014), se refiere a aquellas obligaciones o responsabilidades que son de carácter facultativo, potestativo, voluntario que realiza el tendero de barrio hacia su entorno o hacia las personas que hacen parte del mismo. Ejemplos: destinar recursos de todo tipo a la sociedad, mejorar la calidad de vida, participar en actividades voluntarias y caritativas realizadas en el ámbito en que se realiza la actividad comercial, etc.

\section{Actividades con la comunidad}

Acciones que requieren la participación del tendero de barrio mediante su esfuerzo directo o colaboración directa sin involucrar en todos los casos aporte en dinero. Ejemplos: fiestas para los niños del barrio, arreglo o limpieza de la cuadra, ferias, festividades comunales, etc.

\section{Donaciones y filantropía}

Involucra la donación en especies o en efectivo para ayudar a atender causas sociales, fundaciones, hogares infantiles, de la tercera edad, cuidado del medio ambiente, la parroquia del barrio, grupos sociales específicos, etc.

\section{Servicios sociales}

Constituyen aportes que el tendero de barrio brinda a la comunidad sin cobro alguno de su parte y por fuera de su actividad comercial concreta; ejemplo: aplicar o suministrar medicamentos a los vecinos, brindar información variada, propender por la asociatividad de las personas que desarrollan la misma actividad, ayudar en forma esporádica a la gente del barrio, crear conciencia entre los clientes y vecinos acerca de problemas sociales específicos y de las medidas pertinentes para su prevención, para su control o para su cura (alcoholismo, drogadicción, tabaquismo, daños ambientales, etc.). 


\section{Conceptualización general sobre la responsabilidad social empresarial (RSE)} del tendero de barrio

A partir de la investigación realizada con los distintos informantes de este estudio (Sanclemente, 2012b, 2014) se pudo advertir la presencia en el tendero de barrio de Medellín, de distintas clases de responsabilidad social empresarial las cuales se exhiben en el cuadro $N^{\circ} 1$. Se puede constatar que esta persona lleva a cabo una "responsabilidad social empresarial implícita" ligada a su personalidad y a sus creencias, actitudes y preferencias en este sentido. La operatividad de este concepto es igualmente acometida de manera inconsciente y con débiles niveles de formalización, diferenciándole así de la forma en que practican este concepto las grandes empresas, es decir, de manera evidente y precisa. Fue igualmente posible verificar en este estudio que estos comerciantes son antes que nada "responsables consigo mismos" sobre todo desde el punto de vista económico y que se esfuerzan por sostener su negocio, obtener ingresos suficientes y eventualmente participar con ciertas causas u obligaciones en su entorno. Valoran igualmente la legalidad, el comportamiento justo con el prójimo y evidencian una permanente preocupación por la satisfacción de las necesidades de su mercado.

- Cuadro 1.

\section{Responsabilidad social empresarial del tendero de barrio}

\section{Responsabilidad social empresarial}

1. Responsabilidad social empresarial económica

3. Responsabilidad social empresarial ética

4. Responsabilidad social empresarial legal

5. Responsabilidad social empresarial comercial (clientes)

\section{Elementos}

1. Rentabilidad

2. Fiado

1. Aseo

2. Reciclaje

3. Manipulación de alimentos

4. El evitar contaminación

1. Honesto

2. Justo

3. Influencia sobre el entorno

4. Respeto

1. Cumplimiento obligaciones contractuales

2. Alimentos certificados

3. Formalización/registro del negocio

1. Relaciones con los clientes

2. Precios

3. Producto

4. Satisfacción

5. Servicio

6. Fiado 
Responsabilidad social empresarial

6. Responsabilidad social empresarial (proveedores)

7. Responsabilidad social empresarial voluntaria

\section{Elementos}

1. "Boca-oreja"/voz-a-voz

2. Acuerdos comerciales

1. Actividades con la comunidad

2. Donaciones y filantropía 3. Servicios sociales

Fuente: elaboración propia (Sanclemente, 2012b, 2014).

Varios elementos que son constitutivos de la responsabilidad social empresarial igualmente le generan preferencias ante su mercado al tendero de barrio. Es estimado, considerado y preferido ante otras alternativas comerciales debido principalmente a toda una serie de comportamientos que muestra en su actividad diaria y que sin él proponérselo le va generando cierta estimación en sus públicos, y ello incide en sus resultados financieros. Este comportamiento que se menciona no es estratégico ni intencionado, es esporádico y está básicamente apoyado en los valores, en supuestos, en creencias y actitudes de esta persona y de esa manera es que es racionalizado por ella y puesto en práctica, es como una forma de él transformarse para transferir valor a la comunidad en la que se desenvuelve con su oficio.

Adquiere reputación cuando se comporta de manera responsable con su negocio y a pesar de que otorga crédito a sus clientes (fiado), muchas veces sin tener conciencia acerca del alcance del manejo de este tema en su rentabilidad, lo realiza basado en la confianza con la gente y buscando prestar un servicio a la clientela, puesto que ella es su "razón de ser" y de esta manera también puede lograr su fidelidad. Cuando muestra una conducta inclinada a la preservación del medio ambiente a través de labores de reciclaje y aseo en su negocio y en la cuadra en que trabaja, eso es valorado igualmente por los demás y le ocasiona preferencias entre su público, lo cual incide en sus ventas y en su crecimiento. Se ha encontrado igualmente en esta indagación que, el hecho de tener productos legales, cumplir con determinadas normativas oficiales en cuanto al expendio de productos (no vender tabaco o licor a menores de edad, por ejemplo) y el procurar adoptar acciones tendientes a su formalización o hallarse dentro de ella en el desempeño de su actividad, esto le procura también un renombre y una consideración por parte de sus públicos, pues entre otras esa situación brinda garantías tanto a consumidores como a proveedores.

Finalmente, y aún en el campo de los componentes de la RSE que procuran reputación, se encontró que la honestidad y el comportamiento ético del tendero inciden en el posicionamiento ante sus proveedores y clientes (ser recto en cuanto a pagos y en cuanto al establecimiento de precios, respectivamente). La responsabilidad que manifiesta este comerciante es inherente a su personalidad, la muestra cuando atiende a sus clientes con los productos adecuados, legales, en buen estado y cuando se esmera por prestar un eficiente servicio procurando guardar las relaciones con ellos durante largo tiempo.

\section{Conclusión}

En la investigación base del presente artículo, los principales hallazgos muestran que el concepto de la responsabilidad social empresarial (RSE) tiene aplicación para los tenderos de barrio y consta de seis tipos distintos y complementarios: económica, legal, ética, voluntaria, ambiental y comercial (Sanclemente, 2012b, 2014). Estos comerciantes fueron sondeados respecto a la importancia que asignaban en su actividad a las diferentes clases de RSE encontradas y la casi totalidad de ellos favorecieron la económica, en cuanto a que su negocio presentara un determinado nivel de rentabilidad adecuado para procurarles unos ingresos específicos 
y suficientes para su subsistencia y la de su familia y para, eventualmente, estar en capacidad de asignar ciertos desembolsos para los demás tipos de RSE.

Fue posible comprobar, en el contexto y persona estudiados, propuestas como las de Spencer \& Rutherfoord (2000) y Jenkins (2006) respecto a que los principales enfoques considerados al examinar las prácticas de pequeñas empresas en el terreno de la RSE son, entre otros aspectos, los de establecer prioridades en temas tales como: el incremento de las ganancias o beneficios, la estabilidad y sostenibilidad como empresa para contar con calidad de vida y el sustento básico, el interés propio al comprometerse en acciones sociales que de manera consciente les procuren beneficio en su actividad comercial, y la de efectuar aportes sociales y/o económicos a la comunidad, incluso más allá de sus propios intereses financieros ${ }^{6}$.

Se constató adicionalmente que las actuaciones desplegadas en este ámbito revisten un carácter informal y no hacen parte de un programa estratégico, con el interés de ganar imagen o reputación en el mercado atendido, el cual podría ser el caso de grandes empresas, que pretenden, complementariamente a estas acciones, incrementar sus resultados financieros acometiendo proyectos de esta naturaleza tal y como lo podrían insinuar algunos autores (Sanclemente, 2012a; Marín y Ruiz, 2008). Se puede concluir en este sentido que los inductores principales de la RSE en su caso, serían, fundamentalmente, "la personalidad, los valores, las actitudes, los principios, los supuestos que este empresario/propietario/comerciante tiene sobre la relación de sus negocios con los diferentes grupos de interés, así como sobre cómo se debe crear y transferir valor en conjunto. Esta especificidad tornaría la RSE que se practica en estos negocios, en una "actividad informal, esporádica, implícita, no planificada" (Barrera y Sanclemente, 2012, p. 197). Otra de las particularidades de la RSE en el tendero de barrio, y que se reflejó en el examen de esta investigación, fue el que estos individuos no cuentan con los recursos financieros indispensables para

6. Muchos de los tenderos de barrio indagados asociaron la RSE a algo intrínseco a su personalidad y a su identidad, algo superior a la simple búsqueda de ganancias en su actividad comercial. emprender acciones en este campo, especialmente cuando las mismas no presentan réditos inminentes (Lepoutre \& Heene, 2006); no obstante lo anterior, las actividades realizadas en este sentido, conscientes 0 instintivas, les generan una reputación favorable, y a su vez, un incremento de sus resultados comerciales. Esta circunstancia orientaría a estas personas a buscar apoyo con los entes públicos 0 privados que los soporten en sus proyectos de RSE7. Este estudio permitió también descubrir que en el caso de estos pequeños distribuidores minoristas es muy valioso, en el tema de la RSE, el poseer un pleno conocimiento de cuáles son los distintos grupos de interés o stakeholders que tienen algún tipo de relación con la actividad que ellos desempeñan. En efecto, varios autores se refieren a la utilización de la teoría de los stakeholders, no solo para que los pequeños empresarios (como el tendero) puedan establecer un "marco de acción" en el campo de la RSE sino también para que distintos entes puedan prestar su colaboración en tal sentido (Jenkins, 2006; Perrini, Russo \& Tencati, 2007; Russo \& Tencati, 2008). En el contexto antioqueño, Fenalco (Federación Nacional de Comerciantes) tiene un programa que se denomina "Fenalco Solidario" que otorga un certificado en RSE a todas aquellas empresas, entidades o personas que realicen proyectos propios o con terceros, enfocados al desarrollo social y al sostenimiento del planeta. También esta misma agremiación desarrolla en la región, mediante su programa "Fenaltiendas", un reconocimiento especial que se denomina "El Tendero Líder" y que busca premiar cada año a un tendero de barrio por su valiosa contribución a la comunidad a la que pertenece.

Teniendo en cuenta que existe un vínculo entre el tema de la RSE y el concepto de los grupos de interés o stakeholders para efectos de manejar de modo adecuado las relaciones con estos públicos y sus correspondientes expectativas en la distribución minorista (Whysall, 2000a, 2000b), se propuso en el marco de este estudio, una matriz de responsabilidades del tendero de barrio ante los eventuales

\footnotetext{
7. Varios clientes en los grupos focales expusieron su opinión acerca de que los tenderos deberían recibir más apoyo de proveedores, de entes públicos y de agremiaciones para poder desarrollar actividades más concretas y valiosas para la comunidad en el tema de la RSE, pues este tema superaba la capacidad individual de esas personas para actuar en ese sentido.
} 
públicos que tendrían relación con su negocio. Esta propuesta vino a complementar uno de los objetivos de la investigación, cual era el de describir las actividades que en materia de RSE desarrolla el tendero, puesto que adicionalmente se pudo plantear una guía orientadora para que esta persona tenga en cuenta los potenciales requerimientos de estos públicos en este aspecto tan neurálgico en los negocios actualmente.

\section{Referencias Bibliográficas}

Arlow, P., \& Ackelsberg, R. (1991). A Small Firm Planning Survey: Business Goals, Social Responsibility, and Financial Performance. Akron Business and Economic Review, 22(2), 161-172.

Arnould, E. J., \& Wallendorf, M. (1994). Market-oriented ethnography: Interpretation building and marketing strategy formulation. Journal of Marketing Research, 31, 484-504.

Barrera, E., y Sanclemente, J.C. (2012). Creación de valor mezclado en las pymes latinoamericanas. En Raufflet, E., Lozano, J. F., Barrera, E. y García, C., (Eds.). Responsabilidad Social Empresarial (pp. 185-200). México: Pearson Educación.

Bhattacharya, C.B., \& Sen, S. (2004). Doing Better at Doing Good: When, Why, and How Consumers Respond to Corporate Social Initiatives. California Management Review, 47(1), 9- 24.

Brown, T. J., \& Dacin, A. (1997). The Company and the product: Corporate Associations and Consumer Product Responses. Journal of Marketing, 61, 68-84.

Burton, B. K., \& Goldsby, M. (2009). Corporate Social Responsibility Orientation, Goals, and Behavior. A Study of Small Business Owners. Business \& Society, 48(1), 88-104.

CCRE. Centro Colombiano de Responsabilidad Empresarial (2006). Línea de Base sobre Responsabilidad Social Empresarial en Colombia. Estudio 2006. CCRE \& Ipsos Napoleón Franco.

Chrisman, J. J., \& Fry, F. L. (1982). Public versus Business Expectations: Two views on social responsibility for small business. Journal of Small Business Management, 19-26.

Chrisman, J. J., \& Archer, R. W. (1984). Small Business Social Responsibility: Some Perceptions and Insights. American Journal of Small Business, IX(2), 46-58.

Corbin, J., \& Strauss, A. (1990). Grounded Theory Research: Procedures, Canons, and Evaluative Criteria. Qualitative Sociology, 13, 3-21.
Giraldo, G. (2008). Responsabilidad Social Empresarial en Antioquia. Revista Universidad EAFIT, 44(149), 38-59.

Glaser, B.G., \& Strauss, A. L. (1967). The Discovery of Grounded Theory. Strategies for Qualitative Research. New York: Aldine de Gruyter.

Handelman, J. M., \& Arnold, S. J. (1999). The Role of Marketing Actions with a Social Dimension: Appeals to the Institutional Environment. Journal of Marketing, 63, 33-48.

Jenkins, H. (2006). Small Business Champions for Corporate Social Responsibility. Journal of Business Ethics, 67, 241-256.

Lepoutre, J., \& Heene, A. (2006). Investigating the Impact of Firm Size on Small Business Social Responsibility: A Critical Review. Journal of Business Ethics, 67, 257-273.

Lichtenstein, D. R., Drumwright, M. E., \& Braig, B. M. (2004). The Effect of Corporate Social Responsibility on Customer Donations to Corporate-Supported Nonprofits. Journal of Marketing, 68, 16-32.

Maignan, I. (2001). Consumers' perceptions of Corporate Social Responsibilities: A Cross-Cultural Comparison. Journal of Business Ethics, 30(1), 57-72.

Maignan, I., \& Ferrell, O.C. (2004). Corporate Social Responsibility and Marketing: An Integrative Framework. Journal of the Academy of Marketing Science, 32(1), 3-19.

Marín, L., y Ruiz, S. (2008). La Evaluación de la Empresa por el consumidor según sus acciones de RSC. Cuadernos de Economía y Dirección de la Empresa, 35, 91-112.

Marín, L., Ruiz, S., \& Rubio A. (2009). The Role of Identity Salience in the Effects of Corporate Social Responsibility on Consumer Behavior. Journal of Business Ethics, 84, 65-78.

Mohr, L. A., \& Webb, D. J. (2005). The Effects of Corporate Social Responsibility and Price on Consumer Responses. The Journal of Consumer Affairs, 39(1), 121-147.

Murillo, D., \& Lozano, J. M. (2006). SMEs and CSR: An Approach to CSR in their Own Words. Journal of Business Ethics. 67: 227-240.

OBRSC. Observatorio de Responsabilidad Social Corporativa y Fundación El Monte (2005). Guía de la Responsabilidad Social Corporativa para las Pymes. Isabel Roser Hernández.

Perrini, F. (2006). SMEs and CSR Theory: Evidence and implications from an Italian Perspective. Journal of Business Ethics, 67: 305-316.

Perrini, F., Russo, A., y Tencati, A. (2007). CSR strategies of SMEs and large firms. Evidence from Italy. Journal of Business Ethics, 74, 285-300.

Pettigrew, S. F. (2000). Ethnography and Grounded Theory: A happy marriage? Advances in Consumer Research, 256-260. 
La Responsabilidad Social Empresarial (RSE) de los pequeños distribuidores minoristas

Rallapalli, K. C., Vitell, S. J., \& Szeinbach, S. (2000). Marketers' norms and personal values: An empirical study of marketing professionals. Journal of Business Ethics, 24, 65-75.

Royo, T. (2007). La Responsabilidad Social Empresarial. Oportunidad de mejora interna de gestión, ventaja competitiva, diferenciación y reputación de medianas y pequeñas empresas. Dyna, 82(8), 445-449.

Runyan, R. C., \& Droge, C. (2008). A categorization of small retailer research streams: What does it portend for future research? Journal of Retailing, 84(1), 77-94.

Russo, A., \& Tencati, A. (2008). Formal vs. Informal CSR Strategies: Evidence from Italian Micro, Small, Mediumsized, and large firms. Journal of Business Ethics, 85: 339-353.

Sanclemente, J. C. (2012a). Marketing y la RSE. Lo social como estrategia de marketing. En Raufflet, E., Lozano, J. F., Barrera, E. y García, C., (Eds.). Responsabilidad Social Empresarial (pp. 145-156). México: Pearson Educación.

Sanclemente, J.C. (2012b). La reputación del tendero de barrio ante su mercado y sus consecuencias (Tesis doctoral). Universidad EAFIT, Medellín.

Sanclemente, J.C. (2014). Los pequeños distribuidores minoristas y su aporte en la realización de negocios inclusivos en Colombia. Revue Interventions économiques. 50/2014, URL: http://interventionseconomiques.revues.org/2301

Sen, S., \& Bhattacharya, C.B. (2001). Does Doing Good Always Lead to Doing Better? Consumer Reactions to Corporate Social Responsibility. Journal of Marketing Research, XXXVIII, 225-243.

Spence, L. J. (2007). CSR and Small Business in a European Policy Context: The Five "C"s of CSR and Small Business Research Agenda 2007. Business and Society Review, 112(4), 533-552.
Spence, L. J., \& Rutherfoord, R. (2000). Social responsibility, profit maximization and the small firm owner-manager. Journal of Small Business and Enterprise Development, 8(2), 126-139.

Strauss, A., y Corbin, J. (2002). Bases de la investigación cualitativa. Técnicas y procedimientos para desarrollar la teoría

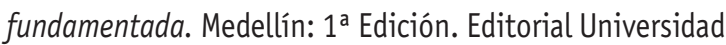
de Antioquia.

Suddaby, R. (2006). From the editors: What Grounded Theory is not. Academy of Management Journal. 49(4), 633-642.

Thompson, J. K. \& Smith, H. L. (1991). Social Responsibility and Small Business: Suggestions for Research. Journal of Small Business Management, 30-44.

Van Auken, P. M., \& Ireland, R. D. (1982). Plain talk about small business social responsibility. Journal of Small Business Management, 1-3.

Vives, A., Corral, A., e Isusi, I. (2005). Responsabilidad Social de la Empresa en las Pymes de Latinoamérica. Subdepartamento de Empresa Privada y Mercados Financieros. Banco Interamericano de Desarrollo.

Wagner, T., Bicen, P., \& Hall, Z. R. (2008). The dark side of retailing: towards a scale of corporate social irresponsibility. International Journal of Retail \& Distribution Management, 36(2), 124-142.

Whysall, P. (2000a). Stakeholder mismanagement in retailing: A british perspective. Journal of Business Ethics, 23, 19-28.

Whysall, P. (2000b). Addressing ethical issues in retailing: a stakeholder perspective. The International Review of Retail, Distribution and Consumer Research, 10(3), 305-318.

Wilson, E. (1980). Social Responsibility of Business: What are the small business perspectives? Journal of Small Business Management, 18(3), 17-24. 